CARNETS OE Carnets de géographes

GÉOGRAPHES.

5 | 2013

Géographie humanimale

\title{
La peur chronique des chiens chez les enfants
}

Une question d'empiétement de l'espace personnel ?

\section{Bénédicte De Villers}

\section{(2) OpenEdition}

Journals

Édition électronique

URL : http://journals.openedition.org/cdg/1064

DOI : $10.4000 /$ cdg. 1064

ISSN : 2107-7266

Éditeur

UMR 245 - CESSMA

Référence électronique

Bénédicte De Villers, «La peur chronique des chiens chez les enfants», Carnets de géographes [En

ligne], 5 | 2013, mis en ligne le 01 janvier 2013, consulté le 19 avril 2019. URL : http://

journals.openedition.org/cdg/1064; DOI : 10.4000/cdg.1064

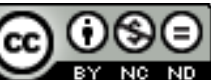

La revue Carnets de géographes est mise à disposition selon les termes de la Licence Creative Commons Attribution - Pas d'Utilisation Commerciale - Pas de Modification 4.0 International. 


\title{
LA PEUR CHRONIQUE DES CHIENS CHEZ LES ENFANTS
}

Une question d'empiétement de l'espace personnel ?

\section{BENEDICTE DE VILLERS}

Docteure en philosophie, Assistante et Doctorante en anthropologie de la communication homme/animal, LASC, Université de Liège benedictedevillers@gmail.com

\section{Résumé}

Dans cet article, est posée la question du partage, par les humains et par les chiens, de l'espace social commun. La phobie des chiens, vécue par des enfants et des adolescents, constitue l'angle par lequel je propose de traiter cette question. Je m'appuie sur une enquête ethnographique menée dans une association qui vient en aide à ces enfants phobiques des chiens. Le but de cette enquête est de décrire les façons dont les enfants vivent leurs rencontres quotidiennes avec des chiens, comme de rendre compte de la façon dont s'est inventé un dispositif thérapeutique qui aborde la peur des chiens sous l'angle du problème de l'espace partagé.

\begin{abstract}
In this article, the question of sharing the common social space by humans and dogs is raised. Phobia of dogs experienced by children and adolescents is the angle by which I propose to address this issue. I draw on an ethnographic fieldwork conducted in an association that helps these children with a phobia of dogs. The purpose of this survey is to describe the ways children live their daily encounters with dogs, as to relate how a therapeutic device that addresses the fear of dogs in terms of shared space problem was invented.
\end{abstract}

Depuis la fin des années 1990, des études scientifiques paraissent, qui insistent sur le nombre de morsures canines sur les enfants. Une étude belge réalisée en 2002 et parue en 2003 dans The Journal of Pediatrics constitue une étape importante dans la mesure où elle montre que le nombre d'enfants victimes de morsures, admis dans des services d'urgences, équivaut à un quart de tous les accidentés de la route et à un tiers des victimes de brûlures domestiques. Qui plus est, cette étude est le fruit d'une enquête pluridisciplinaire qui a mis l'accent sur les conséquences, non seulement physiques, mais aussi psychiques de ces 
morsures. Le risque semble accru, pour ces jeunes victimes, de développer un " syndrome post-traumatique » (PTSD dans la nomenclature anglo-saxonne ; Kahn et al., 2003 ; Peters et al., 2004) qui peut inclure des reviviscences, des troubles du sommeil ou encore le développement d'une phobie des chiens.

Par-delà la question de l'" empreinte psychologique " qu'une morsure de chiens peut laisser sur des enfants, s'ouvre aussi la question de la sensibilité croissante des personnes vis-à-vis de l'occupation, par les chiens, de l'espace social partagé : ainsi, les chiens doivent-ils être tenus en laisse en agglomération, dans les parcs et la plupart des forêts, porter une muselière pour certains d'entre eux, comme si l'intégration des chiens dans nos espaces humanisés devait passer d'abord par un contrôle accru de leur circulation et de leurs mouvements.

Cette question du partage, par les humains et les chiens, de l'espace social commun, j'ai choisi de l'aborder sous l'angle de la peur suscitée par des chiens auprès d'enfants. Pour ce faire, je me fonde sur une expérience professionnelle menée entre 2003 et 2011, au sein d'une association qui se présente comme un "centre éducatif, thérapeutique et d'apprentissage des relations entre les enfants et les chiens ". Ce centre, j'en ai été la fondatrice, accompagnée de psychologues et d'un éleveur/éducateur de chiens. L'un des objectifs que nous poursuivions, en créant un lieu visant de manière générale à "l'harmonisation des relations entre enfants et chiens ", a été notamment de créer un dispositif thérapeutique spécifique, destiné aux enfants qui ont une peur intense et chronique des chiens. À partir de 2008, j'ai entamé un travail ethnographique au sein de cette association. Le but de cette enquête de terrain était à la fois de cerner la singularité de cette peur intense (par rapport à d'autres peurs enfantines), mais aussi de donner une voix ${ }^{1}$ à des enfants prêts à témoigner de leur façon de vivre "le partage de l'espace social " avec des chiens qui les effraient. II ne s'agissait donc pas, dans cette enquête, de recueillir des éléments pour mesurer l'efficacité ou non de cet abord thérapeutique, mais plutôt de décrire les façons dont les enfants vivent leurs rencontres quotidiennes avec des chiens, et de rendre compte de la façon dont s'est inventé un dispositif thérapeutique qui aborde la peur des chiens sous l'angle du problème de l'espace partagé.

Pour l'heure, sur la base de cette enquête de terrain, je souhaite donner à voir comment les chevauchements de territoires humains et canins sont problématiques pour les enfants qui ont une peur intense et chronique des chiens. Mon propos va s'articuler en quatre parties: d'abord, j'exposerai comment a été recueilli le matériel ethnographique. En un deuxième temps, j'indiquerai pourquoi une approche ethnographique de la peur des chiens me semble intéressante et en quoi elle diffère d'une approche psychologique, fréquemment en quête d'une origine de la peur, elle-même considérée comme symptôme. Troisièmement, j'aborderai la question de savoir comment la phobie des chiens chez les enfants renvoie au problème du partage de l'espace social et, du coup, comment, en tant qu'intervenants dans les séances, nous sommes partis de ce problème spatial pour mettre au point notre dispositif thérapeutique. L'hypothèse serait ainsi que la peur des chiens engage une forme de relation

\footnotetext{
${ }^{1}$ Cf. Beaud \& Weber (2008: 8) : « L'ethnographie a pour vocation originaire de rendre la parole aux humbles, à ceux qui par définition n'ont jamais la parole : tribus isolées en terrain exotique, peuples colonisés (ethnologie traditionnelle), classes dominées ou groupes en voie de disparition dans les sociétés développées (ethnologie chez soi) ».
} 
sans espace et, réciproquement, qu'un traitement possible de cette peur pourrait passer par un travail du corps visant à la réappropriation d'un espace personnel et de relations. En conclusion, je risquerai quelques considérations plus générales sur les façons dont sont censées s'agencer, dans notre société moderne occidentale, les territorialités humaines et canines.

\section{1. Éléments de méthode}

Mon idée est de montrer comment la peur des chiens chez les enfants peut renvoyer au problème du partage de l'espace public et constituer une façon de réagir au sentiment d'être envahi dans son " espace personnel " au sens que Goffman a pu donner à ce concept (Goffman, 1973). Je m'appuierai pour ce faire sur l'étude de 46 enfants ayant une peur intense des chiens. J'ai reçu en consultation certains de ces enfants, ou bien ai été observatrice, appareil photo et carnet de notes à la main, d'un travail mené par d'autres intervenants. A l'époque, pour organiser ces consultations, nous sommes partis de l'idée que la phobie des chiens peut se traiter en s'exposant à l'objet de sa peur de façon contrôlée, progressive et dans un cadre sécurisé (dans une perspective qui trouve ses racines dans la thérapie comportementale et cognitive). Dans la foulée, nous avons choisi, en équipe, des chiens à mettre à contribution dans nos séances. Ceux-ci ont été sélectionnés chiots, socialisés aux autres chiens et aux enfants, puis éduqués et, pour le dire vite, " modelés » pour rester paisibles et stables en face d'enfants phobiques dont la réactivité est fréquemment émoussée. Au sein de l'équipe, nous aimions à répéter que nous voulions des chiens "qui n'avaient peur de rien ", sous-entendant par là que nous voulions avoir avec nous des chiens qui ne réagissent pas au moindre bruit, qui ne se méfient ni des autres chiens, ni des humains quels qu'ils soient, y inclus des personnes qui présenteraient des réactions vives en les voyant.

Les 46 enfants reçus en consultation ont entre 3 et 16 ans et sont issus de milieux sociaux variés. La proportion de filles est la même que celle des garçons. Il est à noter que 12 de ces enfants ont été mordus, ou poursuivis, ou agressés, ou simplement témoins d'une agression par des chiens. Au sens strict, un quart de ces enfants peuvent donc être dits présenter un " syndrome post-traumatique ».

Néanmoins, je fais le choix de regrouper l'ensemble de ces 46 enfants et d'évoquer leur phobie des chiens. Leur peur présente en effet des caractéristiques communes, qui justifient, me semble-t-il, l'usage du mot "phobie ": pour l'ensemble de ces enfants, la peur des chiens est chronique, systématique (1); l'adaptabilité de ces enfants aux situations qui incluent des chiens est perturbée (2); bien souvent, la peur de ces enfants vis-à-vis des chiens qu'ils rencontrent paraît disproportionnée et excessive à l'entourage (3) ; enfin, les capacités d'évitement des chiens par les enfants sont sophistiquées, élaborées, jusqu'à parfois devenir si envahissante qu'elles deviennent difficiles à gérer par les parents ou la famille (4). En conséquence, le terme de phobie me semble approprié, même dans les cas où l'enfant n'aurait pas eu à subir de traumatisme. Dans cette optique, plutôt que de me focaliser sur l'origine de la peur, sur l'événement qui en est la source, j'ai voulu être attentive à la façon dont une personne vit ses rencontres avec les chiens, la façon dont elle 
ressent leur présence. Ici, la question « qu'est-ce qui s'est passé » laisse donc place, dans cet article, à celle de savoir " comment cela se passe-t-il actuellement pour celui qui a peur ».

Lorsque des enfants ne parviennent plus à éviter les chiens dans leur parcours quotidiens, leurs parents se renseignent, par exemple sur internet, pour savoir comment l'enfant peut guérir de sa phobie. Ainsi, par exemple, d'une mère qui prend contact avec moi trois semaines avant que sa fille parte en camp de vacances, elle-même effrayée à l'idée qu'il arrive quelque chose à sa fille, tant la peur des chiens chez celle-ci est forte, pouvant la conduire à être imprudente, à échapper à la vigilance du groupe, à s'enfuir et à traverser n'importe où, n'importe comment. D'un côté, l'on est touché par l'angoisse d'une mère pour sa fille; de l'autre, surpris du délai imparti à la "résolution » de cette phobie intense des chiens. Lors du premier rendez-vous avec la jeune fille, j'explique que l'objectif du traitement thérapeutique, qui sera seulement entamé avant son départ en vacances, est qu'elle puisse commencer à " se débarrasser de l'excès de peur " que traduit son inconfort et sa souffrance, mais qu'elle puisse garder néanmoins « la prudence vis-à-vis des chiens en général ». On le devine, la peur se donne d'ores et déjà à voir à travers différentes nuances, différentes intensités qui, à mon sens, dénotent un enjeu ethnographique que je me propose de clarifier.

\section{Approche ethnographique de la phobie des chiens}

L'approche ethnographique de la peur des chiens chez les enfants peut effectivement mettre en lumière sa variabilité : peur des chiens sans laisse qui courent dans les parcs, peur des chiens de races prétendument dangereuses, peur des petits chiens qui aboient ou à l'allure nerveuse. Il est des peurs qui perturbent le cours ordinaire des actions, d'autres qui pétrifient et paralysent, d'autres encore qui provoquent la fuite, qui font crier, hurler, qui suscitent panique et colère. À quoi il faut ajouter les peurs qui rendent les enfants plus vulnérables: il peut arriver qu'une fuite ou un mauvais geste suscite chez un chien une agression voire une morsure.

Sur la variabilité de ces vécus de peur viennent se greffer une diversité de représentations sociales de la peur, symptomatique à mon sens d'une ambivalence qui entoure la peur des chiens et qui peut se résumer ainsi : avoir peur des chiens est-ce un manque de courage ou une nécessité ?

\section{De la faiblesse à la revendication}

D'un côté, la peur des chiens est en effet fréquemment considérée comme une faiblesse, un manque de courage. Leur peur est traitée avec impatience et agacement par l'entourage. "Fais un effort", "tu as 7 ans maintenant", sont des propos qui sortent ci et là. Je me propose ici de relater des extraits d'une séance avec Madeleine, 13 ans, venue avec son père. Madeleine est une jeune fille polie, timide, qui fait du latin et du grec à l'école. Elle hésite à parler, cherche du regard l'approbation de son père pour avancer ses propos. Un souper festif a été organisé par son mouvement de jeunesse où circulait un chien «qui n'avait plus que trois pattes ». Elle raconte s'être déplacée en fonction de la circulation du chien. Pour elle, il s'agit là d'un progrès. Avant le travail que nous avons entrepris, elle se serait immédiatement réfugiée à la maison. Elle précise que le chien était " quand même grand ", ce que son père conteste. Le ton de celui-ci est vindicatif : 
"C'était un chien à 3 pattes qui cherchait de l'amitié ! Madeleine ne fait pas la différence entre un chien qui marque des signes agressifs et un chien amitieux. Je ne comprends pas. Un chien en liberté, là, je comprends, mais pas là (...) II n'y a pas de raison de se méfier. C'était un demi-chien ! " (octobre 2008)

D'un autre côté, la peur des chiens peut également être revendiquée, quasi brandie comme une nécessité. Ainsi de cette adulte qui se fait fort de dénoncer des relations qu'elle estime " déviantes " entre certains propriétaires et leur animal de compagnie. Elle dit nourrir " une méfiance par rapport au manque de civilité des personnes " qui ont des chiens, et en veut pour preuve cet événement qui l'a marqué au point de "vouloir consulter » : elle roule en vélo et un chien lâché arrive à sa hauteur. Elle a "voulu filer ", explique-t-elle, mais se fait quand même attraper le mollet faute d'avoir été assez rapide.

"I'ai hurlé sur le maître, j'étais furieuse. Et le maître: 'y'a pas l'feu !' Mais c'était bleu! On voyait les crocs à travers le jean! Et le maître, il a parlé d'une lèche! J'étais outrée! » (octobre 2008).

Dans la même perspective, certaines personnes soutiennent qu'il serait inconscient de ne pas au moins se méfier des chiens de races dites dangereuses; pour d'autres, il y aurait chaque année assez de morsures et d'agressions de chiens sur des enfants, à domicile ou ailleurs, pour témoigner soit de la dangerosité intrinsèque des chiens, soit de l'inconscience des propriétaires, ou des deux conjointement.

Ainsi, la peur des chiens se déploie en fonction de représentations individuelles particulières. Ajoutons qu'en fonction de ces représentations, cette peur peut être incorporée, apprise, elle peut aussi se transmettre à d'autres de façon plus ou moins consciente. L'apprentissage de la peur des chiens se décline, dans sa version "raisonnable ", en méfiance ou prudence vis-à-vis d'eux. Mais que l'émotion soit trop forte, qu'elle émeuve de façon trop visible, de manière involontaire et non-contrôlée, voilà qui sera moins bien admis. Ce constat empirique pourrait ainsi venir étayer l'idée que notre culture moderne occidentale privilégie l'illusion d'un contrôle mental sur le vécu de chacun : il conviendrait de se contrôler, de gérer ses émotions et de se maîtriser (Elias, 1975 ; Fassin \& Memmi, 2004).

\section{S'accommoder de sa phobie des chiens}

Dans un contexte de contrôle de soi, la phobie des chiens dès lors "fait tâche ". Et je risquerais l'idée qu'elle peut constituer un "stigmate", une singularité particulièrement apparente, dont on tente de s'accommoder. Goffman analyse les stratégies qu'un sujet peut mettre en place pour " gérer un stigmate " (Goffman, 1975). Effectivement, chez les enfants comme chez les adultes, il est possible de repérer différentes stratégies ou tactiques d'accommodation pour gérer cette "marque " que peut représenter pour eux la peur intense et chronique des chiens. Parmi elles, je citerais l'évitement d'une part, et ce qu'on pourrait nommer la manipulation de l'environnement, d'autre part: Les enfants sont redoutables d'ingéniosité pour éviter de croiser des chiens. Pour cette adolescente, reçue en consultations à l'âge adulte, cela signifiait "plaquer ses petits copains " sans explication aucune, si elle avait dû apprendre que l'un d'eux possédait un chien (carnet 2010).

Pour cet enfant de 4 ans, éviter les chiens est un " art » qui a fini par " épuiser " sa maman qui s'est décidée à consulter : 
"Si Adonis croise un grand chien dans la rue, il faut faire demi-tour. Changer de trottoir ne suffit pas, il faut changer de rue! Adonis crie, hurle, réclame les bras. Je voudrais que les chiens redeviennent vivables pour lui. » (Carnet 2003)

Que la peur constitue une marque ou un stigmate difficile à porter, j'en voudrais encore pour preuve des indications tirées, cette fois, d'observations de comportements manifestés par des propriétaires de chiens, dès lors qu'ils doivent gérer, non pas leur propre peur, mais bien la peur de leur chien. Peut-être que ce petit détour par des observations relatives à des peurs manifestées par des chiens, et difficilement assumées par leur propriétaire, pourraient venir appuyer l'idée que la peur est décidément une émotion mal-aimée dans notre société. J'appuierai cette fois mon propos sur des observations faites lorsque j'étais " maître-chien » et donnais des séances de dressage.

\section{La stigmatisation des chiens peureux}

Sans aller jusqu'à affirmer que les propriétaires des chiens n'admettent que très difficilement la peur de leur chien, j'ai néanmoins pu constater que certains d'entre eux engagent eux aussi des stratégies pour atténuer ce stigmate constitué par l'image du chien peureux ${ }^{2}$. Cette minimisation de la peur du chien peut aller d'une forme d'euphémisation avec humour, au déni, en passant par le silence. J'ai entendu ainsi des propriétaires traiter gentiment leur chien de "couillon ", d'autres prétendre que leur chien "n'aime pas les enfants " (ce qui semble préférable à l'aveu qu'il en a peur et risquerait d'être agressif), ou encore d'autres taire des morsures qui se sont produites auparavant.

Mais une séance de dressage donnée en tant que "maître-chien » en 2010 me semble particulièrement révélatrice des "tactiques d'accommodation " que peuvent élaborer des propriétaires de chiens.

Nous sommes en septembre, sur un terrain de dressage, en présence d'une petite dame et de ses deux grands fils. Au bout de la laisse, un chien croisé noir de type berger. Ce jour-là, il y a du vent et je porte une veste bleue électrique à l'effigie d'une grande marque de nourriture pour chiens, ainsi qu'un bonnet. À mon arrivée, le chien nommé Charly recule, queue immobile et basse. II gronde puis aboie à plusieurs reprises. Comme les propriétaires ne disent rien, je m'étonne. La dame rétorque: "Charly a bien le droit d'avoir des affinités avec certaines personnes plutôt qu'avec d'autres. "

Cette séquence est intéressante à plus d'un titre. Elle met en lumière la difficulté dans laquelle se trouve cette dame à devoir s'accommoder de la peur manifestée par son chien. Partons en effet de l'hypothèse que mon accoutrement (veste voyante et bonnet) ait impressionné Charly et l'ait fait réagir. Voilà qui est difficilement acceptable par sa propriétaire : pas question pour elle d'avoir un chien peureux pour si peu, car elle est venue au dressage pour que son chien fasse du "mordant ", pour qu'il puisse la défendre et la protéger, car elle se sent en insécurité en ville. Reconnaître la méfiance de son chien risquerait donc de mettre en échec son projet. Vues sous un autre angle, la peur du chien et l'agressivité qu'il manifeste à mon endroit peuvent être considérées comme un problème : si ce chien grogne et aboie sur tout ce qui porte un bonnet ou une veste bleue, les risques d'accident sont évidents. Mais ce point-là non plus, la propriétaire de Charly ne peut

\footnotetext{
${ }^{2}$ Sur l'idée que l'identité du "couple " humain/animal subit les conséquences de comportements canins inappropriés pour la sphère publique, comme les morsures, le fait de sauter sur les inconnus, etc. Cf. Sanders, 2003 : 413 et Sanders, 1990.
} 
l'admettre. Selon elle, le fait que son chien soit « un peu agressif » est un gage d'efficacité : il la "défendrait " d'autant mieux en cas d'agression par un tiers. Ainsi, prise dans ces dilemmes, la propriétaire préfère soutenir que c'est le chien lui-même qui n'a pas d'affinité avec moi. Mais cette séquence renvoie encore à un autre point important : les peurs canines peuvent se muer en agressivité quand elles sont laissées à elles-mêmes, quand elles ne sont pas identifiées ou pas traitées. Comme évoqué plus haut, la peur chez les chiens peut aussi $s^{\prime}$ inculquer, se développer, voire se construire de façon consciente ou inconsciente. Elle peut se transformer en " agressivité instrumentalisée ", c'est-à-dire en une forme d'agressivité construite et renforcée - même involontairement - par les humains.

Ceci m'amène à constater, comme précédemment à propos de la phobie des chiens chez les enfants, que la peur chez les chiens se manifeste de façon multiple, avec plus ou moins de force, mais aussi qu'elle renvoie à diverses représentations (notamment une faiblesse avec laquelle on tente, tant bien que mal, de s'arranger). II reste maintenant à articuler le thème de la peur avec celui de l'espace.

\section{Cette peur qui révèle des territorialités humaines et canines}

Comment la phobie des chiens chez les enfants renvoie-t-elle à la question du territoire ? Plus précisément, comment ces enfants se sentent-ils concrètement envahis dans leur territoire personnel par la présence des chiens ? De quelle façon est-ce précisément ce lien entre peur et espace qui a mobilisé l'équipe d'intervenants que nous formions à l'époque pour mettre au point un dispositif d'aide pour ces enfants ? Telles sont les questions que je souhaite aborder à présent.

Pour commencer, et afin de donner du relief aux descriptions des manifestations phobiques d'enfants, qu'on me permette de revenir à la position professionnelle qui était la mienne à l'époque, et qui me faisait passer du statut d'intervenante dans une association dédiée aux relations entre enfants et chiens, à celui de " maître-chien » dans un club de dressage. Ainsi, ai-je procédé en "zig-zag ", observant tour à tour des chiens avec leur propriétaire, et des enfants dans leurs interactions avec des chiens. Or, parmi les chiens qui se rendaient au dressage, des chiens "peureux " ont retenu particulièrement mon attention. C'est à leur propos que je voudrais encore dire quelques mots.

A côté des chiens qui ont peur des bruits soudains, des chiens sensibles à des climats de stress, il y en a qui sont très réceptifs aux formes d'intrusion dans leur " espace personnel " au sens goffmanien, c'est-à-dire aux formes d'approche de cette portion d'espace qui entoure le corps et où toute pénétration sera ressentie comme un empiètement (Goffman, 1973 : 44). En 2006, j'observe ainsi un grand chien d'allure molossoïde, présenté par son jeune propriétaire comme "peureux " vis-à-vis des êtres humains qui lui sont inconnus. Je récolte les clichés du chien et de son maître soucieux, dit-il, de " booster (son) chien, de lui faire confiance, de lui donner du crédit ". Sur une photo, le chien est immobile, les muscles du corps et de la queue sont raides, comme s'il freinait des quatre fers, front vers le bas, regard au sol, un filet de salive coulant de ses babines. Sur une autre, le jeune homme est accroupi à côté de son chien qui a cette fois la tête légèrement dirigée vers l'avant. II lui parle manifestement à l'oreille, un bras posé sur l'encolure du chien qui halète. Sur un 
troisième cliché, le chien est assis, regard horizontal, l'arrière-train collé aux jambes de son maître qui se penche vers lui, qui lui parle et le caresse dans une attitude de soutien, alors qu'en face de ce duo, à environ un mètre de distance, un autre propriétaire et son chien circulent tranquillement.

De ces clichés, je retire que ce grand chien ne souhaite pas être approché par l'objet (ou le sujet) de sa peur, et encore moins touché par lui. C'est bien l'approche ou la distance qui décident soit d'une attitude de recul et de repli chez le chien, ou soit d'une attitude légèrement exploratoire, museau en avant. De façon plus générale, qu'un chien se sente acculé, coincé, et sa peur pourrait se muer en une agressivité qui, soit surgira de façon brusque et soudaine - ce qui fait le côté subit et l'aspect anarchique des morsures par peur soit sera annoncée par des signes de peur et de menaces: recul, grognements, retroussement de babines, aboiements, voire morsures.

En regard de ce type d'observations me paraissent répondre d'autres séquences observées, où ce sont cette fois des enfants qui sont émus, fragilisés et touchés par les propos de certains propriétaires de chiens qui les invitent à venir toucher et caresser leur chien. Comme le disait une femme phobique des chiens, certains maîtres peuvent se transformer en "véritables missionnaires " : ils veulent convaincre que leur chien est gentil, qu'avec leur chien, il n'y a rien à craindre.

Des enfants et des adolescents témoignent également d'une impression d'être touchés dans leur espace personnel, alors que le chien est a priori à une distance socialement acceptable. Curieusement, ces enfants, jeunes et moins jeunes, se sentent menacés par un chien qui les regarde, la gueule ouverte et la langue pendante, assis à plusieurs mètres d'eux. Quoique la posture soit perçue par la plupart des personnes comme "relax", ces enfants expliquent, non pas que le chien a chaud, soif ou qu'il se repose, mais qu'ils ont l'impression que le chien va leur aboyer dessus, qu'il va se jeter sur eux, voire les "dévorer " ${ }^{3}$. Bien entendu, il est possible de proposer une lecture psychologique de ce vécu et de convoquer par exemple l'image archétypale du " grand méchant loup » pour expliquer l'origine de cette peur. Cela dit, tant l'âge de certains jeunes qui relatent un tel vécu, tant le fait qu'ils essayent de faire part d'autre chose que de cette peur archaïque, me paraît, non pas mettre en échec cette lecture psychologique, mais la rendre insuffisante. Pour ma part, je fais le choix d'une lecture que l'on pourrait peut-être qualifier de " géographique " : car tout semble se passer, pour ces enfants et ces adolescents, comme si la distance, entre eux et le chien, ne faisait plus le poids. C'est d'ailleurs ainsi qu'une part non négligeable du traitement thérapeutique de la phobie des chiens, mis sur pied par l'association, a pris son point de départ dans ce vécu subjectif d'absence de distance physique vis-à-vis de l'animal.

\section{Le traitement thérapeutique de la phobie des chiens}

Comme annoncé plus haut, j'ai proposé de rassembler l'ensemble des enfants et adolescents qui avaient peur des chiens - notamment au nom de l'intensité de leur peur et de son aspect chronique - et d'évoquer pour eux quelque chose comme une "phobie " des chiens, en mettant provisoirement entre parenthèses l'origine effective de leur peur (une morsure, une

\footnotetext{
${ }^{3}$ Cet échange de regards qui fragilisent les enfants est fort différent du « regard mutuel » évoqué par Sanders dans le contexte d'interactions amicales entre des propriétaires et leur compagnon à quatre pattes (Sanders, $2003: 415)$.
} 
agression, etc.). En ce sens, mon idée est de décrire comment cela se passe pour ces enfants, plutôt que de poser la question de savoir ce qui s'est ou non réellement produit. Par conséquent, qu'il y ait eu accident ou non, j'aborde le traitement de la phobie de ces enfants à partir de ce qu'ils peuvent dire de leurs vécus, comme à partir des façons dont ils considèrent vivre leur peur. Ce faisant, je m'intéresse à leur perception d'une absence de distance entre eux et le chien.

Un jeune de 12 ans, Fabien, vient en consultation avec son jeune frère et son père. Tous trois arborent un look assez sportif. Son père est agacé par la phobie des chiens de son fils ainé et s'étonne de façon véhémente de l'aspect irrationnel de cette peur. Mais Fabien est incapable de soutenir le regard de la chienne qui est avec moi, assise et détendue (comme en témoigne le fait qu'elle s'appuie sur ma jambe alors que je me tiens à ses côté). Fabien craint d'être mordu au visage, même si le chien est loin (4-5 mètres), assis et tenu en laisse.

On le voit, cette phobie des chiens se déploie dans des situations banales. Elle relève d'un régime d'interaction avec les chiens qui n'a rien d' "extra-ordinaire " (Servais, 2012). Pourtant, cet adolescent sur-interprète chaque indice émanant du corps de l'animal (notamment si celui-ci baille, le regarde ou halète), il produit un excès de significations qu'il croit provenir de l'animal lui-même. En ce sens, la phobie produit un "effet d'agrandissement " ou un " effet-loupe " (Remy, 2009) sur les façons dont les humains et les chiens peuvent «mal s'entendre ». Cette idée s'inscrit, je crois, dans la perspective de V\&C Servais (2009) qui montrent que «le malentendu est la structure même de la communication, au sens où la compréhension est un cas particulier du malentendu et non pas l'inverse. Une conception ordinaire de la communication fait de la compréhension un échange de messages envoyés et reçus. On suppose qu'elle est réussie si le message reçu est " identique " à celui qui a été envoyé, si aucun écart ni aucune altérité ne se glisse dans le processus. Dans cette perspective, il n'y a pas de désaccord fondamental ni de malentendu structurel, mais simplement des cas de "mauvaise compréhension ", qui sont susceptibles d'être corrigés par des arguments plus convaincants et/ou plus contraignants. Dans une tout autre optique, faire du malentendu quelque chose de structurel dans la communication revient à prendre en compte, d'emblée, l'altérité des sujets en situation d'échanges et à accepter qu'autrui ne comprenne pas nécessairement, loin s'en faut, ce que le premier a voulu signifier. Dans ce cas, la communication ne repose pas d'abord et essentiellement sur l'envoi et la réception d'un message qui, dans des conditions idéales, pourra être intégralement décodé et compris, mais elle suppose plutôt des manières irréductiblement différentes d'organiser des relations au monde. Du même coup, l'essentiel du propos de cet adolescent ne réside pas pour moi dans le fait qu'il commet une erreur d'interprétation du comportement du chien ; plus important à mes yeux est la difficulté qu'il éprouve, et qu'il a bien du mal à partager avec autrui ${ }^{4}$, à vivre une interaction qui suppose un échange de regards avec un chien, une interaction qui signifie pour lui l'abolition de l'espace qui le sépare de l'animal, ainsi qu'une mise en danger.

En tant qu'intervenante, je propose donc à Fabien de bouger doucement son regard de gauche à droite, et de constater qu'il ne se produit rien. Cela constitue pour lui une façon d'exercer une première forme de mobilité par rapport au chien. Au cours des séances, il s'agit d'acquérir certains mouvements, certaines positions en face d'un chien immobile. Pour

\footnotetext{
${ }^{4}$ Je remercie Véronique Servais et ses étudiants d'avoir particulièrement attiré mon attention sur ce point.
} 
lui faciliter la tâche, je demande à ma chienne de se coucher, ce qu'elle fait volontiers, parfois allant jusqu'à s'affaler, yeux mi-clos. En proposant à Fabien d'avancer, au moment où il le souhaite, pas à pas et en enfonçant les pieds dans le sol, vers la chienne et moi, mon but est de découper la distance qui sépare le jeune du chien, de la rendre palpable, bref, de lui donner du poids. II m'arrive aussi de placer des objets entre lui et le chien, par exemple une chaise, un bâton, et nous les énumérons, afin encore de donner de la consistance à la distance. Avec d'autres objets, comme une laisse ou une corde, il est par ailleurs possible de créer des jonctions entre les territoires humain et canin. Comme dans le cas où je me place entre le chien et le jeune, en tenant d'abord la laisse avec celui-ci ; puis en la lâchant mais en me tenant toujours entre les deux; puis en m'éloignant progressivement, laissant le duo s'organiser seul, mais en restant une " bouée de secours ».

Le traitement de la phobie des chiens implique ainsi l'acquisition de certaines « techniques du corps ", de positionnements et de conduites dans une approche graduelle et progressive des chiens - une approche qui se déroule, faut-il le préciser, dans un espace thérapeutique et sécurisé, et avec les chiens de l'association. Ces enchaînements de gestes et de postures sont, comme le dit Mauss, des "engrenages" où se mêlent le corps et le mental, l'un entraînant l'autre, et réciproquement. À force d'entraînements, ces engrenages forment un " habitus" que l'on cherche ensemble à rendre mobilisable dans des situations où il y a effectivement des chiens. Mauss note que ces techniques du corps valent aussi comme " une éducation au sang-froid " ou une technique de "résistance à l'émoi envahissant " (Mauss, 1950: 385). C'est une manière de retarder l'envahissement par la panique, pour permettre des mouvements qui soient en accord avec la situation rencontrée. Ainsi, ces techniques du corps valent comme une étape pour faire reculer l'excès de peur que traduit la phobie, tout en permettant d'être prudent vis-à-vis du chien rencontré. Il ne s'agit donc pas, dans cet abord thérapeutique, de prôner une " morale de l'effort " (Diamantis, 2003), mais plutôt de donner des outils à des enfants qui croisent immanquablement des chiens dans leur vie quotidienne, afin qu'ils puissent retrouver un certain confort de vie.

L'absence de distance évoquée par les enfants et les jeunes phobiques des chiens n'est donc pas une métaphore, une représentation ou une façon de parler. Elle traduit un vécu d'empiètement par les chiens et un sentiment d'envahissement de l'espace propre. Du même coup, le traitement de la peur, dans son aspect technique et corporel, se décline en deux phases essentielles: une première phase où l'enfant apprend à dire "non " à l'empiètement de son espace personnel et à faire respecter le "contour " de celui-ci (Goffman, 1973 : 52). Concrètement, l'enfant peut ainsi apprendre à se protéger d'un chien qui saute sur lui pour dire bonjour, à parer les mouvements d'un chien qui vient le renifler de trop près, à rendre légitime l'exercice d'une mise à distance de chiens qui occupent le même espace qu'eux. Suit une deuxième phase où l'enfant peut apprendre à circuler en présence de chiens, tout en faisant respecter son enveloppe corporelle, dont Goffman disait qu'elle valait comme une territorialité égocentrique minimale et qu'elle ne se superposait pas nécessairement à l'« espace personnel » qui, lui, peut être plus large et revendiqué comme tel.

Les techniques du corps sont ainsi des enchaînements de gestes et/ou de mouvements qui doivent permettre, soit de faire face à un chien, soit de se (con)tenir dans d'éventuelles situations critiques où l'enfant aurait envie de fuir (ce qui constitue une façon pour l'enfant 
de rester "neutre " aux yeux de chiens qui peuvent eux-mêmes être dans une situation de peur, en conflits, etc.), soit de circuler en présence de chiens qui vaquent à leurs propres occupations. Une fois légitimée et exercée la possibilité des enfants phobiques à ne pas se laisser envahir par les chiens, peut-être qu'ils accepteront de se laisser toucher ou de se laisser attendrir par eux. Peut-être auront-ils même envie de les caresser. Quoiqu'il n'y ait là rien d'obligatoire.

\section{Conclusion}

Sur la base d'une expérience professionnelle passée, et d'observations récoltées alors, j'ai tenté de montrer que le sentiment d'être envahi dans l'espace personnel a constitué une pierre angulaire de notre travail avec des enfants phobiques des chiens. C'est en ce sens que j'ai envisagé l'idée que cette phobie peut produire un " effet d'agrandissement " sur la question des territorialités humaine et canine : la peur des chiens chez les enfants (comme d'ailleurs la peur chez les chiens) donnerait à voir " en grand " les façons dont sont censées s'agencer quotidiennement les spatialités respectives des uns et des autres : s'il semble ainsi aller de soi que l'enfant qui a peur doive "prendre sur lui ", "faire un effort ", se contrôler et s'adapter à la présence des chiens (quitte à modifier son territoire), il pourrait en aller différemment pour les chiens : ceux-ci paraissent pouvoir protéger leur "place " (parfois rendue visible par l'usage de panier pour chien ou de couverture), leur " espace personnel » (certains chiens ne se laissent pas approcher ou toucher), leur "territoire " (le chien est, dans ces conditions, considéré comme " un bon gardien »), voire leurs " effets personnels » comme un coussin, des jeux, une gamelle, un os, etc. Ces derniers objets pourraient ainsi être considérés comme des "territoires de la possession ", toujours au sens que Goffman a donné à ces concepts dans Les Relations en public.

Pour rappel, Goffman a croisé les perspectives éthologiques et sociologiques afin d'observer et de décrire la manière dont s'agencent les " territoires du moi » dans la vie quotidienne et les relations en public. Or, du croisement de telles perspectives, j'ai voulu m'inspirer ici pour tenter, modestement, d'illustrer l'ambivalence des relations humaines avec les chiens. En effet, qu'on se souvienne que les chiens ont longtemps été quasi ignorés par l'éthologie du vingtième siècle, dont l'objet était d'étudier les espèces animales dans leur " milieu naturel ». Dans ce contexte, le chien, en tant qu'animal domestique, semblait trop dénaturé pour mériter une étude approfondie. Or, aujourd'hui - il s'agit là d'un curieux renversement des choses -, ce sont précisément des arguments de type éthologiques qui sont utilisés pour justifier certaines formes d'organisation spatiale et sociale : ainsi, le chien est parfois décrit comme " un loup civilisé » (Teroni \& Cattet, 2004), comme un animal vivant encore dans une " meute » dont le propriétaire devrait d'ailleurs être le " chef ». En clair, c'est le concept même de naturalité du chien qui est actuellement convoqué pour justifier des cadres sociaux d'interaction, ainsi que des agencements de territoires. Mais l'aspect problématique de ces cadres est visible dans les propos du père d'une fille de 9 ans, reçue en consultation :

"En fait, les gens choisissent le confort de leur chien plutôt que celui d'un enfant. Nous avons été invités chez des amis qui ont un chien, jeune, depuis à peine 6 mois. J'ai expliqué combien Elise a peur des chiens. Alors ils ont mis le chien derrière une barrière. Mais ça dure une demi-heure, et après, comme le chien pleure, alors ils le lâchent. En fait, ils préfèrent le confort de leur chien à celui d'Elise... Pour elle ça a été des heures de stress! Tout ça, parce que le chien pleure.... » 
Ainsi, j'espère avoir pu indiqué que la phobie des chiens chez les enfants est une clé d'entrée intéressante pour qui se penche sur des phénomènes la plupart du temps implicites d'organisation des relations entre humains et chiens. La peur rend visible des attentes sociales rarement exprimées en tant que telles, comme elle montre l'articulation problématique des notions de nature et de société. In fine, les peurs dénotent des problèmes de territorialités et de frontières entre l'homme et l'animal: des frontières malléables et diversement justifiées selon les circonstances; des territorialités humaines et canines qui se croisent et se chevauchent - ce qui est bien le nœud du problème dans la phobie.

\section{Bibliographie}

BEAUD S. \& WEBER FI. (2008), Guide de l'enquête de terrain, éd. La Découverte. DIAMANTIS I. (2003), Les phobies ou l'impossible séparation, Paris, Aubier-Flammarion. ELIAS N. (1975), La dynamique de l'occident, Paris, Calman-Lévy. FASSIN D. \& MEMMI D. (éds) (2004), Le gouvernement des corps, Paris, éd. de l'EHESS. GOFFMAN E. (1973), La mise en scène de la vie quotidienne. 2. Les relations en public, Paris, Minuit.

GOFFMAN E. (1975), Stigmate. Les usages sociaux des handicaps, Paris, Minuit.

KAHN A., BAUCHE P., LAMOUREUX J. (2003), "Child victims of dog bites treated in emergency departments: a prospective survey ", The Journal of pediatrics, 162, pp. 254-258. MAUSS M. (1950), " Les techniques du corps » (1935), Sociologie et Anthropologie, Paris, PUF.

PETERS V., SOTTIAUX M., APPELBOOM J. \& KAHN A. (2004), " Posttraumatic stress disorder after dog bites in children ", The Journal of pediatrics, 144(1), pp. 121-122. En ligne : http://hdl.handle.net/2013/ULB-DIPOT:oai:dipot.ulb.ac.be:2013/87798 (consulté le 5 avril 2012).

REMY C. (2009), La fin des bêtes. Une ethnographie de la mise à mort des animaux, Economica, coll. Etudes sociologiques.

SANDERS C. R. (1990), "Excusing Tactics: Social Responses to the Public Misbehavior of Companion Animals ", Anthrozoös, 4, pp. 82-90.

SANDERS C. R. (2003), "Actions Speak Louder than Words: Close Relationship between Humans and Nonhuman Animals ", Symbolic Interaction, 26 (3), pp. 405-426.

SERVAIS C. \& SERVAIS V. (2009), "Le malentendu comme structure de la communication ", Questions de communication, 15, pp. 21-49.

SERVAIS V., "Comment diviniser son dauphin. Modèles de relation, régimes d'activité et savoirs anthropo-zoologiques ", DUMAIN A. (éd.), Passions cognitives, sous presse.

TERONI E. \& CATTET J. (2004), Le chien, un loup civilisé, Québec, éd. Le Jour. 